\title{
AUTOMATIC BUILDING EXTRACTION FROM AIRBORNE LIDAR POINT CLOUD BASED ON MEAN SHIFT SEGMENTATION
}

\author{
Z. Hui ${ }^{\text {a*}}$, Y. Hu ${ }^{\text {a }}$ Y.Y. Ziggah ${ }^{\mathrm{a}}$ \\ a Faculty of Information Engineering, China University of Geosciences, Wuhan 430074 - huizhenyang2008@163.com
}

KEY WORDS: Airborne LiDAR, Building extraction, Mean shift, Segmentation

\begin{abstract}
:
Building extraction is an important part for smart city construction. This paper proposes a novel method for automatic building extraction from airborne LiDAR point cloud. In the present study, filtering was first applied to point cloud, which could help obtain elevated points for generating the DTM. The building-candidate points were then obtained by setting a threshold from the DTM. To distinguish the tree points from building points, three constraints, namely, area constraint, point density constraint and root mean square error constraint were applied to the building-candidate points. By comparing with the reference data generated manually, the evaluation result shows that the proposed method could yield a good performance.
\end{abstract}

\section{INTRODUCTION}

\subsection{Introduction}

The Building extraction from airborne LiDAR point cloud has been a research hotsopt for several years. Many researchers have made many contributions on this area. However, there are still some unsolved problems such as the inability of detecting the false plane generated by tree crowns, low detection accuracy, cumbersome process among others . In this paper, a novel automatic building extraction method has been proposed to resolve the above-mentioned problems.

Building extraction from airborne LiDAR point cloud always involves two main steps, namely, filtering and segmentation. Filtering is a key step which has three main effects, such as downsizing the point cloud, generating DTM and identifying elevated objects. Segmentation plays a role in clustering points with similar characteristics which will help distinguish building points from tree points.

\section{METHODS}

\subsection{Pre-processing}

Due to the multi-path errors and errors in the laser range finder, the obtained point cloud always include outliers, especially low outliers, which will have a significant effect on the following steps. Thus, these outliers should be removed firstly. This paper adopted the method proposed by Hui et al. (2016) to denoise the point cloud.

After the denosing step, the point cloud was organized as grid cells. At this step, only the lowest point in each grid cell was involved in processing. This would help to remove tree points and points on walls. Generally, buildings are composed roofs and vertical walls, so no laser beams could penetrate the building. Thus, there are no points inside the building. In contrast, the laser beams could penetrate brunches or leaves of the tree, capturing not only the tree crown but also surface underneath the tree crown. Therefore, if we select the lowest points in grid cells, most points reflected from tree crown could be removed. Moreover, since laser beams always have incidence angles, there are many points reflected from walls, which will have some effects on the following mean shift segmentation. By selecting the lowest points, these wall points could be removed.

\subsection{Filtering}

This paper adopted the filtering algorithm proposed by Hui et al. (2016) to generate the DTM. For each non-ground points, if its elevation is more than the given threshold $T_{h}$, this point will be labeled as building-candidate points. The threshold was defined as Equation (1).

$$
T_{h}=H_{g}+H_{c}
$$

where $\quad H_{g}=$ the corresponding ground height

$H_{c}=$ a height constant

\subsection{Mean shift segmentation}

The mean shift was first applied to image segmentation (Comaniciu \& Meer, 2002). Here we adopted it for segmenting the building-candidate points. The principle of mean shift is trying to get closer to a local maximum by moving along the mean shift vector as shown in Figure 1.

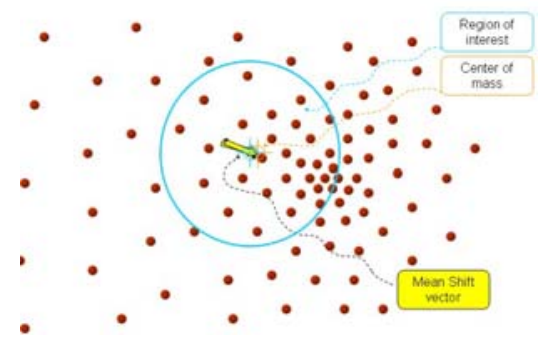

* Corresponding author 
Figure 1. Mean shift sketch map

In this paper, the mean shift vector was defined as Equation (2).

$$
m s(X)=\frac{\sum_{i=1}^{n} X_{i} K^{s}\left(\left\|\frac{X^{s}-X_{i}^{s}}{h^{s}}\right\|^{2}\right) K^{r}\left(\left\|\frac{X^{r}-X_{i}^{r}}{h^{r}}\right\|^{2}\right)}{\sum_{i=1}^{n} K^{s}\left(\left\|\frac{X^{s}-X_{i}^{s}}{h^{s}}\right\|^{2}\right) K^{r}\left(\left\|\frac{X^{r}-X_{i}^{r}}{h^{r}}\right\|^{2}\right)}-
$$

$$
\text { where } \begin{aligned}
n & =\text { the number of points } \\
K^{s} & =\text { horizontal kernel } \\
K^{r} & =\text { vertical kernel } \\
X^{s} & =\text { components of the horizontal vector } \\
X^{r} & =\text { components of the vertical vector }
\end{aligned}
$$

This paper chose uniform as the kernel function considering the rate of convergence and the sensitive of feature. The uniform kernel is defined in Equation (3) as

$$
K(X)= \begin{cases}1 & \text { if }\|X\|<1 \\ 0 & \text { if }\|X\| \geq 1\end{cases}
$$

The mean shift segmentation results are shown in Figure 2(a). It can be found that the building-candidate points were clustered into different segments, including connected components and scattered points. To obtain an accurate building extraction results, three constraints should be applied to the mean shift segments.

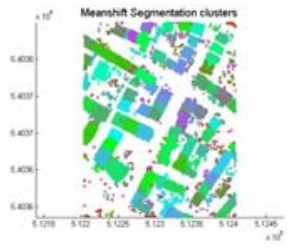

(a)

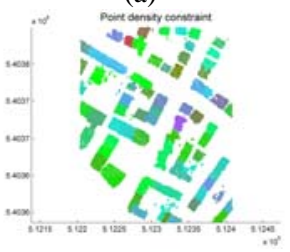

(c)

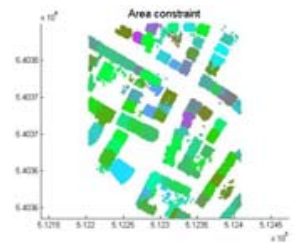

(b)

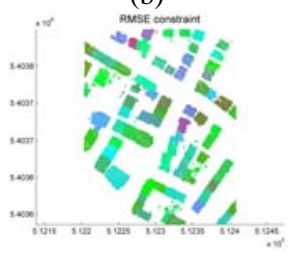

(d)
Figure 2. Result for each step

\subsection{Area, point density and RMSE constraints}

Since buildings are always connected components, their areas are not less than a given threshold. After applying the area constraint, a "clean" result was obtained as shown in Figure 2(b). However, there are still some scattered points, which could be some high trees. To remove these points, point density constraint was applied to the result of last step. By searching the neighbors of each point, if the number of its neighbors is less than a given threshold, this point will be labeled as non-building building points. As mentioned above, some dense tree crowns could generate some false planes. To remove these false planes, root mean square error (RMSE) constraints was applied to the point density constraint result. If RMSE of the segment is more than a given threshold, this segment will be removed. The final result is shown in Figure 2(d).

\section{EVALUATION}

The proposed method was evaluated by comparing the extracted results with the manually corrected results according to the object-based evaluation techniques proposed by Rutzinger et al. (2009). Three indicators defined in Equation (4) were utilized.

$$
\begin{aligned}
& \text { Completeness }=\frac{T P}{T P+F N} \\
& \text { Correctness }=\frac{T P}{T P+F P} \\
& \text { Quality }=\frac{T P}{T P+F P+F N}
\end{aligned}
$$

The evaluated results are shown in Table 1.

\begin{tabular}{|c|c|c|}
\hline Completeness & Correctness & Quality \\
\hline $93 \%$ & $95 \%$ & $91 \%$ \\
\hline
\end{tabular}

Table 1. Margin settings for A4 size paper

\section{CONCLUSION}

On the basis of the results obtained (Figure 2), a conclusion could be drawn that the proposed method can detect buildings effectively and yield a good performance even though there are many dense tree crowns surrounding the buildings.

\section{REFERENCES}

Comaniciu, D., 2002. Mean shift: A robust approach toward feature space analysis. IEEE transactions on Pattern Analysis and Machine Intelligence, 24, pp. 603-619.

Hui, Z., 2016. An Improved Morphological Algorithm for Filtering Airborne LiDAR Point Cloud Based on Multi-Level Kriging Interpolation, Remote Sensing, 8(1), pp. 35.

Rutzinger, M., 2009. A comparisonof evaluation techniques for building extraction fromairborne laser scanning. IEEE Journal of Selected Topics inApplied Earth Observations and Remote Sensing, 2(1), pp. 11-20. 